\section{Urgencias neurológicas y modelos organizativos}

\section{Neurological emergencies and models of care}

\section{Sr. Editor:}

He leído con gran interés el artículo recientemente publicado en su Revista de V. Álvaro Soto y colaboradores que lleva por título "Tiempos de atención de urgencias neurológicas en un hospital regional de alta complejidad"' y querría hacer algunos comentarios sobre el mismo.

En la introducción los autores hacen hincapié en la prevalencia de las urgencias neurológicas y en la distinción con aquellas que representan un riesgo vital, especialmente las urgencias neurovasculares. Igualmente se referencian las recomendaciones de la Sociedad Española de Neurología publicadas en el año 2002 sobre la demora en el tiempo de atención por parte del especialista en Neurología en los pacientes con urgencias neurológicas. Esas recomendaciones hay que valorarlas en su contexto, puesto que ha transcurrido más de una década desde entonces.

En la actualidad hay una coincidencia por parte de la comunidad científica de la importancia de organizar la atención urgente priorizando las patologías tiempo dependientes que, en el caso de las urgencias neurológicas, son las urgencias neurovasculares; como es conocido, son las que producen una mayor morbi-mortalidad y las que con más frecuencia requieren una intervención por parte del neurólogo ${ }^{2}$. Para agilizar la asistencia de estos pacientes se han organizados los llamados código ictus intra y extrahospitalario ${ }^{3}$, buscando la coordinación de todos los profesionales que intervienen en la asistencia para garantizar un diagnóstico precoz y un tratamiento en el lugar, tiempo y por el profesional más adecuados. Esta forma de organización de la atención urgente no es exclusiva de las urgencias cerebrovasculares, sino que es común en otras patologías tiempo dependientes, como el síndrome coronario agudo ${ }^{4} \mathrm{o}$ la sepsis ${ }^{5}$. Sobre el ictus, hay evidencia de que el lugar más adecuado para su manejo son las unidades de ictus ${ }^{6}$, cuya gestión corresponde a médicos neurólogos. Pero antes de que un paciente llegue a una unidad de ictus, intervienen otros profesionales: técnicos de transporte, médicos y enfermeras de los sistemas de emergencias y médicos y enfermeras de los Servicios de Urgencias Hospitalarios (SUH). Por otro lado, no todos los pacientes tendrán próximo un hospital de alta complejidad, por lo que es imprescindible prestar la atención precoz en el lugar más próximo al evento agudo y, en caso de que sea necesario, disponer de la posibilidad de traslado rápido al centro donde se realizará el tratamiento específico. La telemedicina ${ }^{3}$ permite la valoración por parte de profesionales expertos desde los centros de referencia para dar soporte a los profesionales de los sistemas de emergencias extrahospitalarios o bien a profesionales de hospitales en los que no hay neurólogo de guardia. El código ictus es, pues, un modelo de atención basado en protocolos de actuación transversales, esto es, que implican a todos los profesionales que intervienen en el proceso asistencial. En este modelo de atención el neurólogo tiene un papel fundamental, pero ya no es el tiempo que tarde en ser valorado por éste desde su llegada al SUH lo que condicionará el resultado final. De hecho, tras una activación de código ictus, el neurólogo del centro hospitalario receptor es conocedor del tiempo estimado de llegada del paciente por lo que la demora en la atención por parte de este es prácticamente inexistente. En cualquier caso, no hay que olvidar que el tiempo de atención extrahospitalaria y de traslado y la recepción por parte de los profesionales médicos y enfermeros del Servicio de Urgencias hospitalario es igualmente trascendente.

Igualmente, las urgencias neurológicas no vasculares precisan para su atención de la competencia específica en urgencias neurológicas, que no solo tienen los médicos especialistas en Neurología, sino también los médicos de Urgencias convenientemente formados. En estos casos, el especialista en Neurología puede ser consultado por el médico de Urgencias, pero tampoco sería éste el tiempo que determine el pronóstico del paciente, siempre que la primera atención haya sido la adecuada.

El modelo de atención directa de pacientes desde el triaje $\mathrm{e}^{7}$ por parte de especialistas de las diferentes especialidades médicas y quirúrgicas, sigue presente en muchos centros, también en España, donde en cada comunidad autónoma la organización del sistema sanitario es competencia del gobierno autonómico, lo que ocasiona una heterogeneidad organizativa en los Sistemas de Emergencias y en los Servicios de Urgencias hospitalarios ${ }^{8}$.

No obstante, a nuestro juicio, el modelo de atención urgente hospitalaria que mejor responde a las necesidades de los ciudadanos debería basarse en un triaje realizado por profesionales con un adecuado perfil competencial ${ }^{9}$, con una distribución de pacientes priorizando los que tienen mayor riesgo y recayendo la responsabilidad del proceso de atención urgente en profesionales propios del SUH apoyados por especialistas de diferentes especialidades en función de la cartera de servicios de cada centro. Este modelo de atención debe de acompañarse de una organización de la atención extra e intrahospitalaria a partir de los códigos en aquellas patologías tiempo dependientes.

Por último, la innovación ${ }^{10}$ en la organización de los SUH es deseable, sobre todo teniendo en cuenta que no hay un único modelo organizativo con suficiente evidencia científica. Ahora bien, es preciso hacer hincapié en la importancia de cómo se miden las intervenciones realizadas, sobre todo cuando se trata de cambios en la 
organización de la atención que, en ocasiones, resulta difícil demostrar que han supuesto una verdadera mejora. En el caso que nos ocupa, el indicador utilizado para medir los resultados de la intervención es el tiempo de atención por parte del neurólogo (indicador de proceso), pero no sabemos si esa mejora en la demora en la atención se tradujo en una mejora en los indicadores de resultado, como son la mortalidad, complicaciones, capacidad de resolución, o en la satisfacción de los profesionales y de los ciudadanos. Es lógico que uno intuya que esa mejora en el tiempo de atención debe traducirse en una mejora en los resultados, pero no se puede inferir sin evaluarlo.

\section{Antonio Juan Pastor ${ }^{1}$ Instituto Catalán de la Salud. Barcelona, España.}

\section{Referencias}

1. Soto A, Gladys I, Morales G, Vega C, Colinas MB, Canales P, et al. Tiempos de atención de urgencias neurológicas en un hospital regional de alta complejidad. Rev Med Chile 2018; 146: 885-9.

2. Gómez Ibáñez Q, Irimia P, Martínez-Vila E. Urgencias neurológicas y guardias de Neurología. Anal Sis San Navarra vol.31 supl.1 Pamplona 2008.

3. Rodríguez-Castro E, Vázquez-Lima MJ, Rodríguez-Yáñez M, Verde L, Castillo J. Plan de Asistencia al Ictus en Galicia: telemedicina para un tratamiento hospitalario multidisciplinar, precoz y universal del ictus agudo. Emergencias 2018; 30: 54-61.

4. Borrayo-Sánchez G, Rosas-Peralta M, Pérez-Rodríguez G,
Ramírez-Árias E, Almeida-Gutiérrez E, Arriaga-Dávila JJ. Acute myocardial infarction with ST-segment elevation: Code I. Rev Med Inst Mex Seguro Soc 2018; 56 (1): 26-37.

5. Ferreras JM, Arribas B, Sarrat MA, García-Noaín A, Caudevilla A, Colás C, et al. Evaluación de los resultados antes y después de la implantación del Código Sepsis en Aragón. Emergencias 2017; 29: 154-60.

6. Masjuán J, Álvarez-Sabín J, Arias S, Blanco M, de Felipe A, Escudero I, et al. Urgent stroke care in hospitals with a stroke unit. Quick Project. Rev Neurol 2016; 62 (7): 303-10.

7. Fontova A, Suñer R. La importancia del triaje en los servicios de urgencias. Emergencias 2017; 29: 204-10.

8. Juan A. Servicios de urgencias hospitalarios en España: ¿diferentes comunidades, diferentes organizaciones?. Emergencias 2017; 29: 369-70.

9. Martínez-Segura E, Lleixà M, Salvadó $\mathrm{T}$, Solà E, Adell M, Chanovas Manel R, et al. Perfil competencial en los profesionales de triaje de los servicios de urgencias hospitalarios. Emergencias 2017; 29: 173-7.

10. Lobón L, Anderson P. Innovación en Medicina de Urgencias y Emergencias: cinco aspectos organizativos que podrían cambiar nuestra práctica. Emergencias 2017; 29: 61-4.

Conflicto de intereses: El autor declara que no ha recibido financiación alguna para la elaboración del manuscrito y que no existe ningún conflicto de interés.

Correspondencia a:

Antonio Juan Pastor, PhD.

Instituto Catalán de la Salud.

Calle Ferrocarril no 1-3 Esc C $3^{\circ} 2^{\mathrm{a}}$. Botigues de Sitges 08860

Barcelona, España.

a.juanpastor@gmail.com
Los autores aludidos ofrecieron la siguiente réplica:

\section{Urgencias neurológicas y modelos organizativos. Réplica}

\section{Neurological emergencies and models of care. Reply}

\section{Señor Editor:}

El objetivo del estudio citado fue mostrar una experiencia de atención por neurólogos en un servicio de urgencia (SU) de un hospital público regional ${ }^{1}$. Principalmente en lo que respecta al grado de cumplimiento de la recomendación de evaluación por neurólogo(a) dentro de la primera hora desde el inicio de los síntomas $^{2}$. Idealmente, en urgencias neurológicas de riesgo vital (status epilepticus, hemorragia subaracnoidea), la evaluación debiera realizarse dentro de $15 \mathrm{~min}$ desde el ingreso $^{2}$. En nuestro estudio, solo $27,1 \%$ de los pacientes fue evaluado en menos de una hora desde su llegada al
SU, con una diferencia significativa entre Urgencias Vasculares y No Vasculares $(22,6 \text { versus } 36 \%)^{1}$. No sólo las urgencias neurovasculares son tiempo-dependientes, lo son también el status epilepticus y la meningoencefalitis infecciosas. La evaluación rápida ligada a un manejo certero y precoz se asocia a mejores desenlaces funcionales.

La participación de neurólogos en los servicios de urgencia públicos en Chile, tanto presencial como de llamada es reciente, particularmente en hospitales regionales como el nuestro. Recién en 2011 en el Hospital Dr. Hernán Henríquez Aravena (HHHA) de Temuco-Chile se incluyó la atención neurológica en horario hábil, y a partir de julio de 2013 se inició la asistencia presencial en la urgencia en modalidad 24/7 .

Concordamos con el Dr. Pastor en que no se puede concluir que la disminución en el tiempo de evaluación por neurólogo se asocia directamente a un mejor resultado funcional en nuestros pacientes. Pero, en particular para los pacientes con Ataque Cerebrovascular (ACV), 
la disminución del tiempo entre la llegada del paciente y la evaluación por neurólogo(a) se traduce en una reducción del tiempo entre el inicio de los síntomas y el inicio de las terapias de reperfusión (trombolisis endovenosa, trombectomía mecánica). Se ha estimado que por cada 15 min de disminución del retraso para la trombolisis se obtiene $4 \%$ de aumento en la probabilidad de un buen desenlace funcional ${ }^{3}$. Por otra parte, la evaluación precoz por neurólogo(a) permite decidir rápidamente la estrategia de reperfusión más adecuada para los pacientes que consultan dentro de la ventana terapéutica.

Creemos firmemente que los pacientes con patologías neurológicas tiempo-dependiente se benefician al ser evaluados por un especialista en neurología lo antes posible. En la actualidad es bajo el número de neurólogos en los servicios de urgencia de los hospitales públicos chilenos, en este sentido concordamos con el Dr. Pastor en que no todos los pacientes con patologías neurológicas pueden ser evaluados presencialmente por neurólogos. En este contexto, la Teleneurología, en particular el TeleACV, puede suplir en parte el diagnóstico y orientación terapéutica de estos pacientes.

Si bien la recomendación de evaluación de precoz por neurólogo(a) realizada por la OMS data de 2002. Ha sido refrendada por el Plan Estratégico Nacional para el Tratamiento Integral de las Enfermedades Neurológicas en España en sus versiones 2002 y $2016^{2,4}$. Asimismo, la guía chilena de $\mathrm{ACV}$ isquémico agudo señala que "la evaluación inicial de la persona con sospecha de un ACV debe hacerse sin demora"

La existencia de guardias de neurología se asocia a una disminución significativa del tiempo de atención de los pacientes. En un estudio español multicéntrico, el tiempo promedio puerta-neurólogo fue de $34,5 \mathrm{mi}-$ nutos, en los hospitales que contaban con guardia de neurología, comparado con 607,3 min en los hospitales que no tenían este recurso ${ }^{6}$. Si bien valoramos el aporte de la Teleneurología, consideramos que no reemplaza a la evaluación presencial de pacientes con patologías neurológicas agudas. Por lo anterior sugerimos la existencia de neurólogos presenciales en todos los SU de los hospitales que tengan una población asignada entre 500 mil y un millón de habitantes².
Dr. Álvaro Soto Venegas ${ }^{1}$

${ }^{1}$ Departamento de Especialidades Médicas, Facultad de Medicina, Universidad de La Frontera.

Temuco, Chile

\section{Referencias}

1. Soto A, Morales G, Vega C, Colinas MB, Canales P, Contreras $\mathrm{D}$. Tiempos de atención de urgencias neurológicas en un hospital regional de alta complejidad. Rev Med Chile 2018; 146: 885-9.

2. Sociedad Española de Neurología. Plan estratégico nacional para el tratamiento integral de las enfermedades neurológicas. Mayo 2002. Disponible en: http://www.sen.es/pdf/ planacional.pdf [Consultado el 28 de octubre de 2017].

3. Saver JL, Fonarow GC, Smith EE, Reeves MJ, Grau-Sepulveda MV, Pan W, et al. Time to Treatment With Intravenous Tissue Plasminogen Activator and Outcome From Acute Ischemic Stroke. JAMA 2013; 309 (23): 2480-8.

4. Sociedad Española de Neurología 2016. Plan Estratégico Nacional para el Tratamiento Integral de las Enfermedades Neurológicas "PENTIEN II". Disponible en: https://issuu. com/senmadrid/docs/pentien_ii [Consultado el 31 de marzo de 2019].

5. Ministerio de Salud de Chile. Guía clínica AUGE. Accidente Cerebrovascular Isquémico en personas de 15 años y más. Serie de las guías clínicas de MINSAL, 2013. Disponible en: http://web.minsal.cl/portal/url/item/7222754637e58646e04001011f014e64.pdf [Consultado el 28 de octubre de 2017].

6. Castillo J, Vivancos-Mora J, en nombre de los investigadores del registro EPICES. Tiempos de llegada al hospital y hasta la atención neurológica de pacientes con ictus agudo. Análisis de un registro multicéntrico nacional: registro EPICES (II). Rev Neurol 2012; 54: 461-7.

Correspondencia a:

Álvaro Soto Venegas

Departamento de Especialidades Médicas, Facultad de Medicina,

Universidad de La Frontera.

Francisco Salazar \# 01145. Temuco, Chile.

alvaro.soto@ufrontera.cl 